\title{
Measuring Left Ventricular Ejection Time using Under-the-Mattress Sensor
}

\author{
Itamar Sela ${ }^{1}$, Zvika Shinar ${ }^{1}$, Kouhyar Tavakolian ${ }^{2}$ \\ ${ }^{1}$ EarlySense, LTD, Ramat Gan, Israel \\ ${ }^{2}$ Biomedical Physiology and Kinesiology Department, Simon Fraser University, Vancouver, Canada
}

\begin{abstract}
Internal bleeding may be hard to recognize before hypovolemic shock onset. Early detection of a shortening in LVET may expedite medical care and increase survival rate. The current experiment aimed to demonstrate the ability to measure LVET using analysis of the cardioballistic effect measured with a piezoelectric sensor (PE) placed under the mattress. 10 participants were asked to lie supine on a bed with their Lower Body enclosed in a Negative-Pressure (LBNP) chamber. Study's protocol included a baseline phase followed by a gradient reduction of the pressure inside the chamber from -20 to $-60 \mathrm{mmHg}$ with $10 \mathrm{mmHg}$ steps and returning to normal at the end. Blood pressure was measured and a trained echocardiographer performed echo measurements (EC-LVET) to create reference datasets. The PE system was used to measure heart rate, respiration and later extract ES-LVET throughout the test. Comparing the PE's measurements with the reference showed a correlation of $0.76(p<0.001)$. Simple criteria for hypovolemia detection performed better based on LVET than on other vital signs. The current study's results support the ability to use the PE system under-the-mattress for early detection of hemorrhage.
\end{abstract}

\section{Introduction}

During internal hemorrhage (GI bleeding), physiological regulatory mechanisms are effective in maintaining blood pressure (BP) at a stable level with mild blood loss (up to about 15\% of the central blood volume), whereas heart rate (HR) changes during blood loss are complex and dependent on the stage of hypovolemia [1, 2]. Successful surgical treatment of the causes of hemorrhage and decreased levels of later complications are both associated with intervention early in the shock process, demonstrating the importance of recognizing bleeding as early as possible. However, as the BP and HR and the respiratory rate (RR) systems are reflexively and centrally regulated [3] it is often the case that the drop in BP due to the blood loss takes time, which delays the diagnosis of the hemorrhage [4] which in turn may put the patient at risk.

Previous studies indicated that the (LVET) may serve as a marker for a cardiac stress as it tends to be affected faster and earlier by the bleeding as compared to the HR and BP $[5,6,7]$. The LVET shortening is considered to be a result of a decline in stroke volume caused by the progressive loss of blood. Using LVET might lead to early detection of GI bleeding and possibly save patients that will likely progress to hemorrhagic shock and require lifesaving interventions.

The Lower Body Negative Pressure (LBNP) technique is used to simulate internal bleeding by changing the distribution of the blood within the body. It effectively decreases central blood volume in a similar fashion as acute hemorrhage [8]. Moreover, recently it was used to demonstrate the reduction of LVET as the negative pressure increases [5, 9].

The current study aimed to investigate the ability to measure the LVET using an under-the-mattress PE sensor. This type of sensor was previously used to continuously monitor patients in several hospital settings to reduce mortal events of patients and shorten the response time of the medical team for cardio and respiratory emergencies $[10,11]$. Using the PE sensor to identify reduction in the value of LVET could help to early alert the medical staff for a suspicion of an internal bleeding among patients at risk such as individuals after accident or surgical operation.

\section{Method}

Participants: 10 Individuals took part in the study, including six males and four females. Their age was 26.9 \pm 5.22. All participants reported good health with no history of medical or cardiovascular disorders that may affect or influence their safety and the study's results. Informed consent approved by the Simon Fraser University Ethics committee was obtained prior to each individual's participation in the study.

Apparatus: An LBNP chamber was used to remove blood from circulation by pulling it toward the legs, in controlled steps. Participants were asked to lie supine on a bed with their legs in the chamber (Figure 1). An elastic belt was attached to the participants' iliac crest to seal the entrance of the chamber and to form an isolated air surrounding within the chamber. The monitoring of the cardio-vascular system was conducted using an under-the- 
mattress PE sensor that was located below the sternum. An Echo-cardiograph data was collected using Vivid-q system (General Electric).

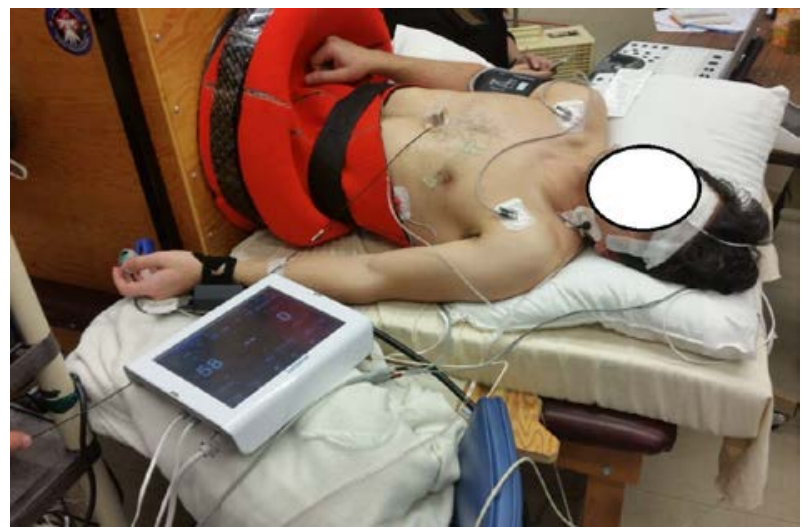

Figure 1. The experiment setup. The participants were laying supine with their lower body enclosed in the LBNP chamber. Information regarding their cardiovascular activity was collected using an under-the-mattress sensor.

Protocol: The protocol included seven steps, each step had a time length of 12 minutes. In every step, the pressure within the chamber was changed as follows:

1. Pre-Baseline: the pressure level within the chamber is equal to the room pressure.

2. P20: reducing the pressure within the chamber by 20mmhg ( $<15 \%$ volume loss- Class I hemorrhage).

3. P30: reducing the pressure within the chamber to be $30 \mathrm{mmHg}$ below room pressure ( $15 \%$ volume loss).

4. P40: reducing the pressure within the chamber to be $40 \mathrm{mmHg}$ below room pressure ( $20 \%$ volume lossClass II hemorrhage).

5. P50: reducing the pressure within the chamber to be $50 \mathrm{mmHg}$ below room pressure ( $23 \%$ volume loss).

6. P60: reducing the pressure within the chamber to be $60 \mathrm{mmHg}$ below room pressure ( $>25 \%$ volume loss).

7. Post-Baseline- pressure is back to normal.

The total timeline of the protocol was 84 minutes. However, participants were allowed to terminate the experiment at any time they felt uncomfortable or the nurse identified pre-syncope signs. In these cases, the pressure was immediately changed back to normal, and protocol jumped to Post-Baseline.

Data collection and feature extraction: Two different LVET datasets were extracted. Echo-Cardiograph LVET (EC-LVET) was computed by the echo-Cardiograph technician. For each participant, three minutes and nine minutes after the beginning of each of the steps, an average LVET value from three heart beats was calculated. The second dataset (ES-LVET) was extracted from the PE signal by a proprietary algorithm developed by Earlysense LTD at the same time areas in which the EC-LVET measurements were collected. In addition, an ongoing HR was collected and BP was measured every 3 minutes.

Data analysis: Pearson correlation analysis was used to test for statistical relationship between EC-LVET and ESLVET.

In the second phase of the data analysis, the ES-LVET, $\mathrm{BP}, \mathrm{HR}$, and the RR were used to develop an alert for a suspected internal bleeding condition. The assumption was that in cases where the ES-LVET or the BP are significantly decreased or $\mathrm{HR}$ or RR are significantly increased, as compared to the Pre-Baseline phase, a suspected internal bleeding condition occurred. Thus, for each of the biomarkers, the minimum value of the PreBaseline minus the standard deviation of the Pre-Baseline (for ES-LVET and BP) or higher than the maximum value of the Pre-Baseline plus the standard deviation of the PreBaseline (for HR and RR) was set as a threshold. Once the mean value of the phase was lower (ES-LVET and BP) or higher (HR and RR) than the threshold, the phase was considered to be the time in which the suspected internal bleeding alert is triggered.

\section{Results}

As was expected, the change in the pressure within the chamber affected the LVET in a linear manner, thus, an increase in the negative pressure was accompanied with a faster LVET. This was observed in both EC-LVET and ESLVET. Figure 1 presents a typical example of the two different LVET measurements throughout the process of an ongoing increasing of LBNP. In this specific example, both EC-LVET and ES-LVET were around 310 milliseconds during the pre-baseline phase. Both LVET measurements decreased as the negative pressure increased. The two LVET measurements reached a minimum value of around 200 milliseconds in Phase P60. The return of the negative pressure to normal at PostBaseline phase led to an increase in LVET values as well.

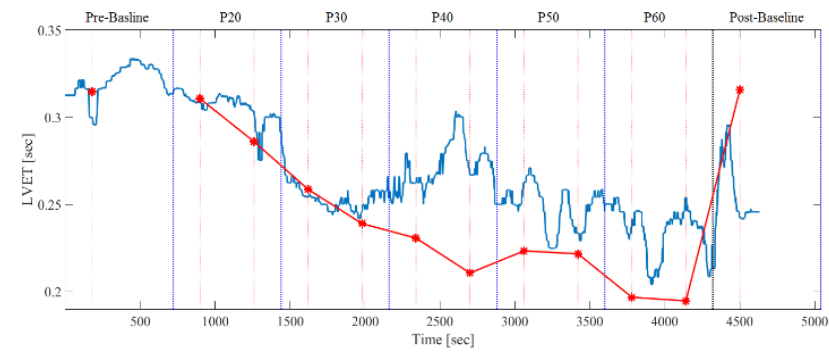

Figure 2. ES-LVET (blue) and EC-LVET (red) of a representative participant. A reduction in the value of LVET was observed in both datasets together with the increase of the negative pressure.

This linear relationship between the negative pressure and LVET was found in most of the individuals that took 
part in this study. Figure 2 presents the mean and standard deviation of the ES-LVET and EC-LVET in each of the protocol's phases for the 10 participants. As can be seen in the graph, both LVET measurements started with a value of around 300 milliseconds. The gradient increased negative pressure was accompanied with a reduction of both LVETs throughout all phases. Both LVET measurement returned to the value of 300 milliseconds during the Post-Baseline phase, in which the pressure within the chamber returned to normal. A Pearson correlation analysis revealed a significant positive correlation between EC-LVET and ES-LVET $(r=0.76$, $\mathrm{p}<0.01)$.

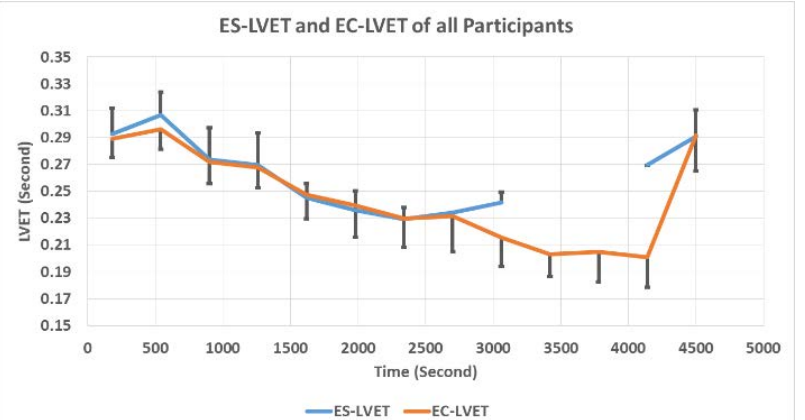

Figure 3. The mean (and standard deviation) of the ECLVET and EC-LVET for all participants during the process of pressure reduction. Note that for the participants that the protocol was terminated before protocol ending, their LVET performance during the Post-Baseline phase is presented at the last time point in the presented graph.

Not all participants were able to finish the entire timeline of the protocol- 84 minutes. Overall, six out of the 10 participants were asked or have been required to terminate the protocol before the full planned time (three participants were asked to stop the protocol at P40, one participant at P50 and additional 2 at P60). This led to a reduced number of successful ES-LVET as well as ECLVET measurements as the protocol advanced.

The ongoing reduction of the ES-LVET enabled to develop an alert for a suspected internal bleeding. The alert was defined as the phase in which the mean value of a phase's ES-LVET was lower than the minimum value of ES-LVET of the Pre-baseline phase minus the Prebaseline's standard deviation. The same technique was applied to the measurements of the BP, the HR, and RR. As for the ES-LVET, the alert positively identified 8 out of the 10 participants to be suspected as suffering from an internal bleeding, 6 of them were identified at the P20 phase and 2 at P30 (Table 1). The results of the average time in which the alerts were produced (based on the order of the different phases) revealed that the ES-LVET alert was the fastest as compared to BP, HR, and RR. Furthermore, the ES-LVET alert was more accurate compared to the other measures as it alerted in 8 out 10 participants, while the $\mathrm{BP}, \mathrm{HR}$, and RR were triggered in 7,6 , and 2 of the participants only, respectively.

Table 1. The suspected internal bleeding alert. For each participant, the phase in which the Pre-Baseline threshold was crossed for ES-LVET, HR, RR, and BP was measured.

\begin{tabular}{c|cccc} 
Participant & ES-LVET & $B P$ & $H R$ & $R R$ \\
\hline 1 & & 40 & 50 & 60 \\
2 & 30 & & 40 & 60 \\
3 & 20 & 20 & 30 & \\
4 & 20 & 20 & 30 & \\
5 & 20 & 20 & & \\
6 & 20 & & & \\
7 & 20 & 40 & & \\
8 & 30 & 50 & 40 & \\
9 & 20 & 50 & 50 & \\
10 & 22.50 & 34.29 & 40.00 & 60 \\
mean & 4.33 & 12.94 & 8.16 & - \\
Standard- & & & &
\end{tabular}

\section{Discussion}

The main purpose of this study was to demonstrate the ability to measure LVET by using a contact free under-themattress sensor. By successfully measuring LVET, a possible identification of an internal bleeding condition may be allowed. The results of the current study provide promising evidence for such an ability. Moreover, the proposed computed ES-LVET biomarker may allow to develop an LVET based monitor that will be used in different hospital departments where an internal bleeding is considered to be a risk, but nevertheless currently not under ongoing monitoring.

Previous studies were able to show the ability to measure LVET using different technologies including Photoplethysmographic Pulse Oximeter [6, 12], or PE sensors [5, 9]. However, unlike the setting in the current research, in most of the studies, the sensor is attached to the participant's body, a setup that produced a signal with a relatively high signal to noise ratio. The results of the current study provide evidence for the ability to extract LVET from a contact free under-the-mattress PE sensor. The analysis showed a reduction in ES-LVET that accompanied the increasing of the negative pressure, in similar to the performance of EC-LVET. Moreover, a significant correlation between the ES-LVET and ECLVET across all participants was found. The fact that it is possible to measure LVET indirectly increases the functionality of such a technique and may help to facilitate it in different medical situations.

The current study results support the notion that under progressive hemorrhage, LVET may be changed prior to BP or HR response $[6,7]$. As can be seen in Table 1, ESLVET indicated for a suspected internal bleeding before 
the other collected measurements. Nevertheless, the data in Figure 3 reveal that the used technique to extract ESLVET failed to produce data in later phases within the protocol. This may be explained by two factors: the first is the reduced number of participants that were able to continue the protocol up until its P40-P60 as six out of the 10 participants were asked to terminate the protocol prior to its planned ending. In addition, it may be the case that although the remained four participants continued to the protocol's end point, they also felt discomfort and suffered from the beginning of the cardiovascular stress. The uneasy feeling have led participants to restlessness that altered heart and body behavior and significantly decreased signal to noise ratio.

\section{Conclusion}

In conclusion, this study demonstrated the ability to measure LVET using a contact free under-the-mattress sensor. This may help measuring LVET continuously in many different medical setups where internal bleeding may be a risk. The shortening of the LVET during early stages of internal hemorrhage, as compared to BP and HR can be used for better patient safety and enhanced outcome. Further work is required to determine false alert rate in non-bleeding patients. In addition, an adaptive filtering may be required to extract LVET during near-shock conditions. Future work will also be needed to address the question whether this method is valid for hypovolemia caused by dehydration.

\section{References}

[1] R. E. Little, E. Kirkman, P. Driscoll, J. Hanson and K. Mackway-Jones, "Preventable deaths after injury: why are the traditional 'vital' signs poor indicators of blood loss?," Journal of Accident and Emergency Medicine, vol. 12, pp. 1-14, 1995.

[2] S. McGee, W. Avernethy and D. Simel, "The rational clinical examination. Is this patient hypovolemic?," JAMA, pp. 1022-1029, 1999.

[3] K. S. Derek and K. J. Shoemaker, "Hypovolemia and neurovascular control during orthostatic stress," American Journal of Physiology Heart Circulatory physiology, vol. 282, no. 2, pp. 645-655, 2002.

[4] G. Gutierrez, D. H. Reines and M. E. Wulf-Gutierrez, "Clinical review: Hemorrhagic shock," Clinical Care, vol. 8, pp. 373-381, 2004.

[5] K. Tavakolian, G. A. Dumont, G. Houlton and A. P. Blaber, "Precordial Vibrations Provide Noninvasive Detection of Early-Stage Hemorrhage," SHOCK, vol. 41, no. 2, pp. 91-96, 2014.

[6] G. Chan, P. Middleton, B. Celler, L. Wang and N. Lovell, "Detecting Change in Left Ventricular Ejection Time During Head-Up Tilt-Induced
Progressive Central Hypovolemia Using a Finger Photoplethysmographic Pulse Oximetry Wave Form," Journal of Trauma-Injury Infection \& Critical Care, vol. 64, no. 2, pp. 390-397, 2008.

[7] T. Geeraerts, P. Albaladejo, A. D. Declère, J. Duranteau, J.-P. Sales and D. Benhamou, "Decrease in Left Ventricular Ejection Time on Digital Arterial Waveform during Simulated Hypovolemia in Normal Humans," Journal of Trauma-Injury Infection \& Critical Care, vol. 56, no. 4, pp. 845-849, 2004.

[8] W. H. Cooke, K. L. Ryan and V. H. Convertino, "Lower body negative pressure as a model to study progression to acute hemorrhagic shock in humans," Journal of Applied Physiology, vol. 96, no. 4, pp. 1249-1261, 2004.

[9] K Tavakolian, G Dumont, A Blaber. Analysis of seismocardiogram capability for trending stroke volume changes. A lower body negative pressure study. Computing in Cardiology 2012;39:733-736.

[10] H. Brown, J. Terrence, P. Vasquez, D. W. Bates and E. Zimlichman, "Continuous Monitoring in an Inpatient Medical-Surgical Unit: A Controlled Clinical Trial," The American Journal of Medicine, vol. 127, pp. 226-232, 2014.

[11] T. Klap and Z. Shinar, "Using Piezoelectric Sensor for Continuous-Contact-Free Monitoring of Heart and Respiration Rates in Real-Life Hospital Settings," Computing in Cardiology, vol. 40, pp. 671-674, 2013.

[12] P. M. Middleton, G. S. Chan, E. O'lone, E. Steel, R. Carroll, B. G. Celler and N. H. Lovell, "Changes in left ventricular ejection time and pulse transit time derived from finger photoplethysmogram and electrocardiogram during moderate haemorrhage," Clinical Physiology and Functional Imaging, vol. 29, no. 3, pp. 163-169, 2009.

[13] A. T. Cote, A. A. Philips, S. S. Bredin and D. E. Warburton, "A questionable association of stroke volume and arterial pulse pressure under gravitational stress," Trauma, vol. 72, pp. 708-712, 2012.

[14] R. W. Stafford, W. S. Harris and A. M. Weissler, "Left Ventricular Systolic Time Intervals as Indices of Postural Circulatory Stress in Man," Ciculation, vol. 41, no. 3, pp. 485-492, 1970.

Address for correspondence

Itamar Sela, Earlysense, Z'abotinsky 7, Ramat Gan, Israel. itamar.sela@earlysense.com 